\section{(2) OPEN ACCESS}

\title{
Inline perfusion mapping provides insights into the disease mechanism in hypertrophic cardiomyopathy
}

\author{
Claudia Camaioni, ${ }^{1}$ Kristopher D Knott (1) , 1,2 Joao B Augusto, ${ }^{1,2}$ Andreas Seraphim, ${ }_{1,2}^{1,2}$ \\ Stefania Rosmini, ${ }^{1}$ Fabrizio Ricci, ${ }^{3}$ Redha Boubertakh, ${ }^{1,4}$ Hui Xue, ${ }^{5}$ Rebecca Hughes, ${ }^{1,2}$ \\ Gaby Captur, ${ }^{1,2}$ Luis Rocha Lopes, ${ }^{1,2}$ Louise Anne Elizabeth Brown —0 , ${ }^{6}$ \\ Charlotte Manisty, ${ }^{1,2}$ Steffen Erhard Petersen, ${ }^{1,4}$ Sven Plein, ${ }^{6}$ Peter Kellman, ${ }^{5}$ \\ Saidi A Mohiddin, ${ }^{1}$ James C Moon ${ }^{1,2}$
}

\begin{abstract}
- Additional material is published online only. To view please visit the journal online (http://dx.doi.org/10.1136/ heartjnl-2019-315848).
\end{abstract}

${ }^{1}$ Advanced Cardiac Imaging, Barts Health NHS Trust, London, UK

${ }^{2}$ Institute of Cardiovascular Science, University College London, London, UK

${ }^{3}$ University of Chieti-Pescara, Chieti, Italy

${ }^{4}$ The William Harvey Research Institute, Queen Mary University of London, London, UK

${ }^{5}$ National Institutes of Health, Bethesda, Maryland, USA ${ }^{6}$ Department of Biomedical Imaging Science, University of Leeds, Leeds, UK

\section{Correspondence to}

Dr James C Moon, University College London, London WC1E 6BT, UK; j.moon@ucl.ac.uk

CC and KDK contributed equally.

Received 15 August 2019 Revised 29 October 2019 Accepted 5 November 2019 Published Online First 10 December 2019

\section{ABSTRACT}

Objective In patients with hypertrophic

cardiomyopathy (HCM), the role of small vessel disease and myocardial perfusion remains incompletely understood and data on absolute myocardial blood flow ( $\mathrm{MBF}, \mathrm{mL} / \mathrm{g} / \mathrm{min}$ ) are scarce. We measured MBF using cardiovascular magnetic resonance fully quantitative perfusion mapping to determine the relationship between perfusion, hypertrophy and late gadolinium enhancement (LGE) in HCM.

Methods 101 patients with HCM with unobstructed epicardial coronary arteries and 30 controls (with matched cardiovascular risk factors) underwent pixelwise perfusion mapping during adenosine stress and rest. Stress, rest MBF and the myocardial perfusion reserve (MPR, ratio of stress to rest) were calculated globally and segmentally and then associated with segmental wall thickness and LGE.

Results In HCM, 79\% had a perfusion defect on clinical read. Stress MBF and MPR were reduced compared with controls (mean \pm SD $1.63 \pm 0.60$ vs $2.30 \pm 0.64 \mathrm{~mL} / \mathrm{g} / \mathrm{min}$ $p<0.0001$ and $2.21 \pm 0.87$ vs $2.90 \pm 0.90, p=0.0003$, respectively). Globally, stress MBF fell with increasing indexed left ventricle mass ( $R^{2}$ for the model 0.186 , $p=0.036$ ) and segmentally with increasing wall thickness and LGE (both $\mathrm{p}<0.0001$ ). In $21 \%$ of patients with HCM MBF was lower during stress than rest $(M P R<1)$ in at least one myocardial segment, a phenomenon which was predominantly subendocardial. Apparently normal HCM segments (normal wall thickness, no LGE) had reduced stress MBF and MPR compared with controls (mean \pm SD $1.88 \pm 0.81 \mathrm{~mL} / \mathrm{g} / \mathrm{min}$ vs $2.32 \pm 0.78 \mathrm{~mL} / \mathrm{g} / \mathrm{min}, \mathrm{p}<0.0001$ ). Conclusions Microvascular dysfunction is common in HCM and associated with hypertrophy and LGE. Perfusion can fall during vasodilator stress and is abnormal even in apparently normal myocardium suggesting it may be an early disease marker.

\section{INTRODUCTION}

Hypertrophic cardiomyopathy (HCM) affects 1 in 500 people and is characterised clinically by unexplained hypertrophy and genetically by mutations in genes encoding (primarily) sarcomeric proteins. ${ }^{12}$ The histological features are myocyte disarray, left ( \pm right) ventricular hypertrophy (LVH), small vessel disease and fibrosis, but how these features develop and relate to adverse outcomes is poorly understood.

Advanced cardiac imaging with echocardiography, myocardial perfusion scintigraphy (MPS), cardiovascular magnetic resonance (CMR) and positron emission tomography (PET) can measure hypertrophy, microvascular dysfunction and fibrosis. However, quantification of these processes is not well integrated into clinical care and this may partially explain our lack of progress on developing disease-modifying therapies.

Ischaemia in HCM is likely a key disease pathway. Chest pain is frequent, ischaemic ECG changes are common ${ }^{3}$ and sudden cardiac death (SCD) is relatively more common during exercise. ${ }^{4}$ Several mechanisms may contribute to ischaemia in HCM including small vessel abnormalities, demand-supply mismatch due to hypertrophy, reduced perfusion pressure related to shortened diastolic time, high diastolic pressure, left ventricular outflow tract (LVOT) obstruction and possibly myocardial bridging. ${ }^{5-8}$

Microvascular dysfunction in HCM was first studied by nuclear medicine techniques, demonstrating perfusion impairment even in apparently minimally affected segments, ${ }^{9}$ with ischaemia correlating with hypertrophy and poor outcomes. ${ }^{1011} \mathrm{CMR}$ has demonstrated evidence of coronary microvascular flow impairment, ${ }^{12}$ finding inverse correlations between perfusion and both hypertrophy and fibrosis. ${ }^{13} 14$ However, the quantification techniques used in these studies have been highly labour intensive in terms of both image acquisition and analysis. Consequently, quantitative perfusion has been less actively explored than scar as a candidate risk factor for SCD and has been outside of the realm of clinical care.

Recent advances in CMR perfusion mapping now permit high-resolution, pixel-wise myocardial blood flow (MBF) quantification automatically and inline at the scanner (figures 1 and 2) ${ }^{15}$ using the Gadgetron software framework. ${ }^{16}$ In suspected ischaemic heart disease the technique has been validated using PET, angiography and invasive fractional flow reserve (FFR). ${ }^{17-19}$ Perfusion mapping has also provided insights into the disease process in Fabry disease. ${ }^{20} \mathrm{CMR}$ has further advantages as it does not use ionising radiation, has higher spatial 

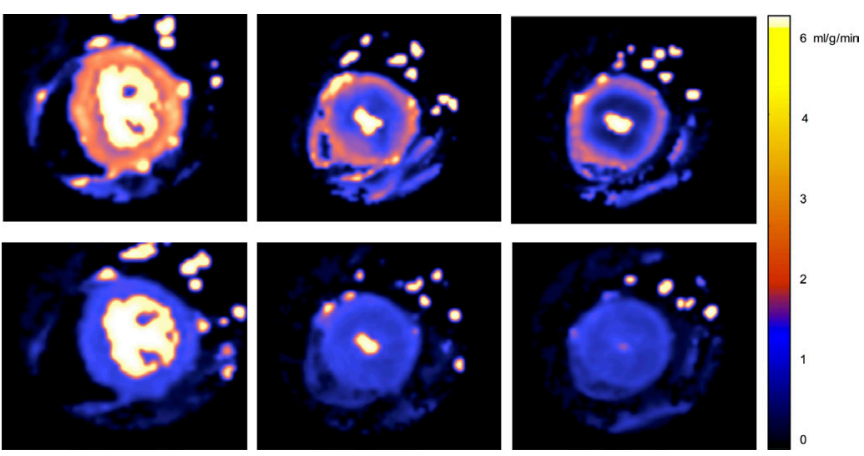

Figure 1 Perfusion maps. Base, mid and apical left ventricular slices (left to right) at peak stress (top) and rest (bottom) in a patient with apical hypertrophic cardiomyopathy. During stress, a circumferential mid to apical perfusion defect is observed, more severe at the apex, particularly in the endocardial layer where the stress myocardial blood flow (MBF) is lower than the rest. Stress MBF values: basal $1.51 \mathrm{~mL} / \mathrm{g} /$ min, mid-ventricular $0.82 \mathrm{~mL} / \mathrm{g} / \mathrm{min}$ and apical $0.53 \mathrm{~mL} / \mathrm{g} / \mathrm{min}$. Rest $M B F$ values: basal $0.93 \mathrm{~mL} / \mathrm{g} / \mathrm{min}$, mid-ventricular $0.79 \mathrm{~mL} / \mathrm{g} / \mathrm{min}$ and apical $0.77 \mathrm{~mL} / \mathrm{g} / \mathrm{min}$.

resolution than other imaging modalities and is becoming more widely available.

We sought to retrospectively quantify myocardial perfusion in clinically referred patients with HCM to better understand the relationship between perfusion, hypertrophy and LGE in the disease process. We hypothesised that perfusion in HCM would be related to markers of disease severity such as hypertrophy and LGE and that impaired perfusion may precede hypertrophy in some myocardial segments suggesting it is an early marker of disease.

\section{METHODS}

\section{Study design and population}

Patients with HCM referred for stress CMR as part of standard clinical care at Barts Heart Centre (London, UK) were enrolled between June 2016 and June 2019. The diagnosis of HCM had been made previously based on conventional diagnostic criteria according to the European Society of Cardiology guidelines. ${ }^{2}$

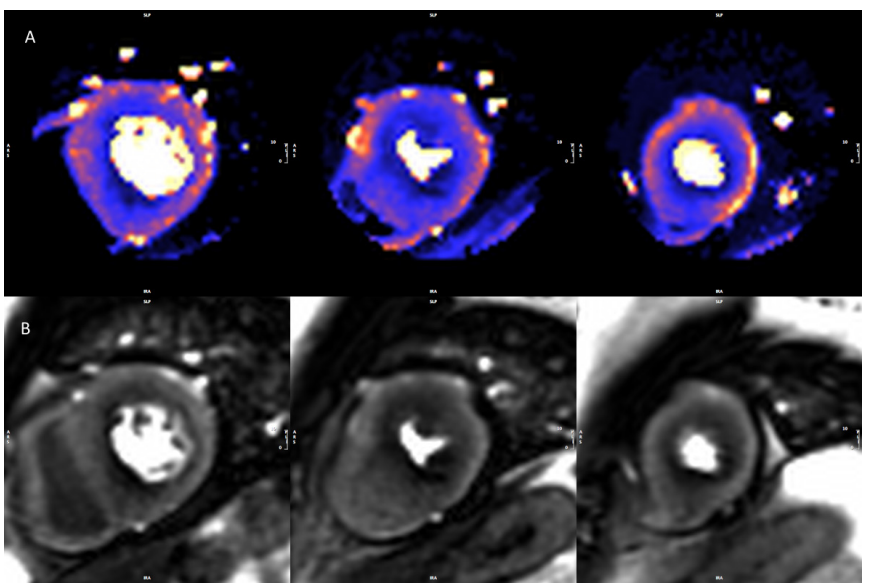

Figure 2 Stress perfusion maps and raw perfusion images. Example of a set of perfusion maps (A) and raw perfusion images (B) from a patient with hypertrophic cardiomyopathy. The perfusion defect is appreciated on visual read but the full extent of hypoperfusion is more readily seen on the perfusion maps. Global stress myocardial blood flow (MBF) is $1.38 \mathrm{~mL} / \mathrm{g} / \mathrm{min}$.
Patients were excluded if the they had epicardial coronary artery disease, defined as $>50 \%$ diameter stenosis in a major coronary artery, by either invasive coronary angiography $(56 \%)$ or CT (44\%) within 3 months of CMR and compared with age, gender, body surface area (BSA) and cardiovascular risk factor matched controls.

Controls included a matched chest pain cohort referred for perfusion CMR. These were patients referred with cardiovascular risk factors (excluding history of myocardial infarction) and atypical chest but otherwise normal CMR (normal structure, function, no perfusion defect and no LGE). This cohort was used to control for other risk factors of microvascular dysfunction which might impair perfusion in patients with HCM independent of the HCM disease process. Study exclusion criteria were contraindications to CMR, adenosine or gadolinium. This study was performed in accordance with the principles of the Declaration of Helsinki and all participants gave written informed consent (217 671 and/or 14/EE/0007).

\section{Image acquisition}

CMR was performed using either a Magnetom Aera 1.5T or Prisma 3.0T system (Siemens Healthineers, Erlangen, Germany). A standard CMR protocol was used including cine images, stress and rest perfusion and late gadolinium enhancement. ${ }^{21}$ All subjects abstained from caffeine for at least 12 hours. Adenosine was infused for $4 \mathrm{~min}$ at $140 \mu \mathrm{g} / \mathrm{kg} / \mathrm{min}$ (increased to $175 \mu \mathrm{g} / \mathrm{kg}$ / min if there was no heart rate response and symptoms). At peak vasodilator stress a gadolinium-based contrast agent (Dotarem, Guerbet, Paris, France) was injected at a dose of $0.05 \mathrm{mmol} / \mathrm{kg}$ at a rate of $4 \mathrm{~mL} / \mathrm{s}$. Three short axis slices (base, mid and apex) were acquired during the first pass of contrast (60 measurements). The acquisition was repeated at rest, with the short axis cine stack acquired between stress and rest.

Perfusion mapping was performed automatically and inline as previously described. ${ }^{15}$ In brief, this was a single-bolus, dualsequence technique with a balanced steady-state free precession (bSSFP) pulse sequence readout. LGE images were acquired in long axis and short axis using a free-breathing bright blood single-shot bSSFP sequence with phase-sensitive inversion recovery reconstruction and motion correction. Sequence details are provided in the supplementary appendix.

\section{Image analysis}

Offline analysis was performed using commercial software (cvi42, Circle Cardiovascular Imaging, Canada). The raw perfusion images were scored for the presence or absence of visual perfusion defects. Cine images, LGE and perfusion maps were analysed globally and segmentally according to the American Heart Association (AHA) 17 segment model (minus the apical cap, figure 3). ${ }^{21}$ The maximum end-diastolic wall thickness (excluding papillary muscles) was determined per segment and the maximum wall thickness globally listed. LGE was quantified using the 5 SD technique, where each slice is manually contoured and a region of interest is drawn in the 'remote' myocardium. The amount of LGE was calculated per segment and globally. Subsequently, each segment was scored as having visually confluent, diffuse or no LGE. Global (average across all pixels throughout the myocardium) and segmental MBFs (average of all pixels within each segment) were calculated inline from the perfusion maps (where each pixel encodes MBFs in $\mathrm{mL} / \mathrm{g} / \mathrm{min}$ ). For each slice, the endocardial and epicardial borders were contoured automatically using a machine learning approach (figure 4). The myocardial perfusion reserve (MPR) was calculated as the ratio 

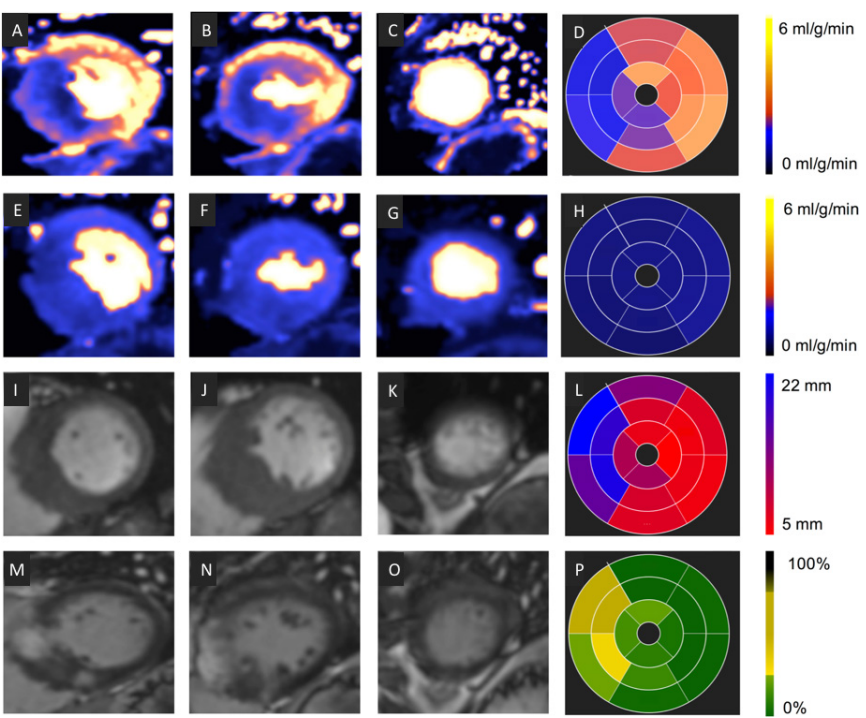

Figure 3 Image analysis. Correlation among stress myocardial blood flow (MBF; A-D), rest MBF (E-H), wall thickness (WT) (I-L) and late gadolinium enhancement (LGE) (M-P). Each row shows a short-axis view (from left, base-mid-apex) and the corresponding 16 segment bullseye. Values are expressed using a specific colour look-up table for $\operatorname{MBF}(D, H), W T(L)$ and LGE where value is percentage of enhanced pixels per segment $(P)$.

of stress to rest MBF. Each segment was also divided into endocardial (inner 50\%) and epicardial (outer 50\%) regions. Each component of the analysis was performed by two independent observers blinded to other CMR parameters.

\section{Statistical analysis}

Statistical analysis was performed using SPSS (V.25, IBM). Categorical data were presented as frequencies and percentage, continuous as mean \pm SD or median and IQR as per normality. The Student's t-test was used for parametric data, Mann-Whitney $U$ test for non-parametric and $\chi^{2}$ for categorical variables. Regression analysis was used to determine the factors associated with perfusion in the HCM subjects. The analysis was performed on both a global (subject level) and segmental level (where the effect of wall thickness and LGE on perfusion was assessed on a segment basis). A mixed effects linear regression model was used at the segmental level to control for within-subject dependency (subjects included as the random effect).

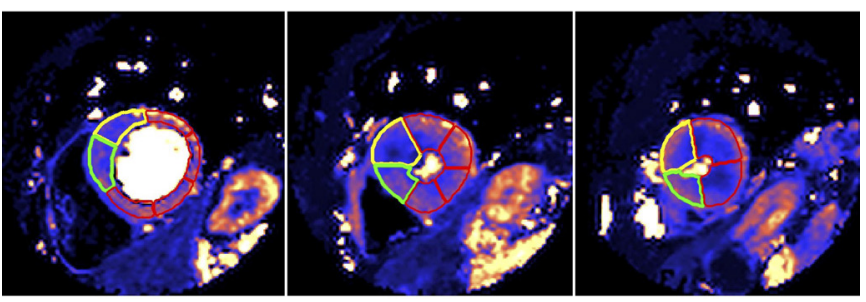

Figure 4 Perfusion map automatic segmentation using machine learning. Basal, mid and apical (left to right) short-axis left ventricular (LV) slices demonstrating automatic segmentation using machine learning. The yellow segments are the starting point for each slice (ie, segments $1,7,13$ ) and the green segments are the second segment in each slice, allowing easy quality control. The remaining segments are contoured red.
Table 1 Characteristics of patients with hypertrophic cardiomyopathy (HCM) and controls

\begin{tabular}{|c|c|c|c|}
\hline & $\begin{array}{l}\text { HCM } \\
n=101\end{array}$ & $\begin{array}{l}\text { Controls } \\
n=30\end{array}$ & $P$ value \\
\hline Age (years) & $49.7 \pm 12.1$ & $51.5 \pm 14.1$ & 0.48 \\
\hline Male, n (\%) & $82(82)$ & $23(77)$ & 0.60 \\
\hline $\mathrm{BSA}\left(\mathrm{m}^{2}\right)$ & $2.03 \pm 0.26$ & $1.97 \pm 0.21$ & 0.32 \\
\hline Diabetes, n (\%) & $17(17)$ & $7(23)$ & 0.43 \\
\hline Hypertension, n (\%) & $44(44)$ & $12(40)$ & 0.83 \\
\hline Dyslipidaemia, n (\%) & $21(21)$ & $7(23)$ & 0.80 \\
\hline LVEDVi $\left(\mathrm{mL} / \mathrm{m}^{2}\right)$ & $72.9 \pm 14.1$ & $77.1 \pm 19.8$ & 0.19 \\
\hline $\operatorname{LVEF}(\%)$ & $74.1 \pm 7.7$ & $64.8 \pm 9.3$ & $<0.001$ \\
\hline LV mass indexed $\left(\mathrm{g} / \mathrm{m}^{2}\right)$ & $87.0 \pm 28.3$ & $55.0 \pm 13.0$ & $<0.001$ \\
\hline LGE, n (\%) & $49(49)$ & $0(0)$ & $<0.001$ \\
\hline Stress MBF (mL/g/min) & $1.62 \pm 0.60$ & $2.31 \pm 0.64$ & $<0.001$ \\
\hline Rest MBF (mL/g/min) & $0.79 \pm 0.24$ & $0.82 \pm 0.25$ & 0.47 \\
\hline
\end{tabular}

Data are presented as mean \pm SD unless stated. $P$ values in bold are statistically significant.

BSA, body surface area; LGE, late gadolinium enhancement; LVEDVi, left ventricle end-diastolic volume indexed for BSA; LVEF, left ventricular ejection fraction; MBF myocardial blood flow.

\section{RESULTS}

\section{Population}

In total, 101 patients with HCM (male $82 \%$, mean age $49.7 \pm 12.1$ ) and 30 patient controls (male $77 \%$, mean age $51.5 \pm 14.1$, $\mathrm{p}=0.60$ and $\mathrm{p}=0.48$, respectively). The HCM clinical phenotype was asymmetric septal hypertrophy in 80 (79.2\%), concentric in $13(12.9 \%)$ and apical or apical predominant in 8 (7.9\%). No patient had undergone septal reduction therapy (myectomy or alcohol ablation) and no patients had a cardiac implantable electronic device. Seventy-one patients $(70.3 \%)$ were scanned at $1.5 \mathrm{~T}$ and $30(29.7 \%)$ were scanned at $3.0 \mathrm{~T}$.

Compared with the control cohort, the patients with HCM had higher indexed left ventricle (LV) mass, maximum wall thickness and ejection fraction (EF) (table 1). There was no difference in end-diastolic volume (EDV). Forty-nine patients with HCM had LGE (48.5\%) and there were perfusion defects in $79(78.2 \%)$ on clinical read. In the HCM group, LVOT obstruction was present under scanning conditions in 17 (16.8\%).

\section{Quantitative perfusion analysis}

Global stress MBF and global MPR were lower in HCM than controls (MBF $1.63 \pm 0.60 \mathrm{~mL} / \mathrm{g} / \mathrm{min}$ vs $2.30 \pm 0.64 \mathrm{~mL} / \mathrm{g} / \mathrm{min}$, $\mathrm{p}<0.001$; MPR 2.21 \pm 0.87 vs $2.90 \pm 0.90, \mathrm{p}<0.001)$ with no difference at rest $(0.79 \pm 0.24$ and $0.82 \pm 0.24, p=0.47)$ (figure 5). Although 78\% of patients with HCM had a perfusion defect on visual analysis, there was no difference between the global stress MBF of those with a perfusion defect and those with no perfusion defect $(1.61 \pm 0.59 \mathrm{~mL} / \mathrm{g} / \mathrm{min}$ vs $1.70 \pm 0.66 \mathrm{~mL} / \mathrm{g} /$ min, $\mathrm{p}=0.522)$. There was no difference in global perfusion in patients scanned at $1.5 \mathrm{~T}$ vs $3 \mathrm{~T}(1.61 \mathrm{~mL} / \mathrm{g} / \mathrm{min}$ vs $1.68 \mathrm{~mL} / \mathrm{g} /$ min, respectively, $\mathrm{p}=0.552$ ).

In HCM, stress MBF was lower in the subendocardium versus subepicardium $(1.38 \pm 0.57 \mathrm{~mL} / \mathrm{g} / \mathrm{min}$ vs $2.32 \pm 0.97 \mathrm{~mL} / \mathrm{g} / \mathrm{min}$, $\mathrm{p}<0.001$ ), but rest not significantly different (figures 1 and 2). This stress developed transmural gradient also occurred in controls $(2.20 \pm 0.61 \mathrm{~mL} / \mathrm{g} / \mathrm{min}$ vs $3.24 \pm 0.94 \mathrm{~mL} / \mathrm{g} / \mathrm{min}$, $\mathrm{p}<0.001)$.

A multivariate linear regression analysis was performed to see the factors contributing to perfusion in patients with HCM on a global (whole heart) basis. Included in the regression were 


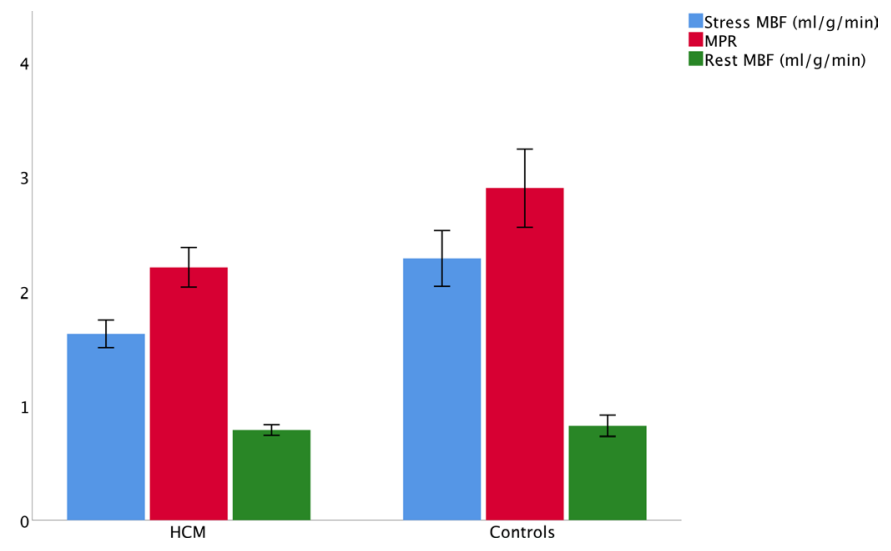

Figure 5 Global perfusion analysis. Differences in stress mean myocardial blood flow (MBF, blue), myocardial perfusion reserve (MPR, red) and rest MBF (green) between patients with hypertrophic cardiomyopathy (HCM) and controls. The bars display the $95 \% \mathrm{Cls}$. Stress MBF and MPR were lower in HCM than controls $(1.63 \pm 0.60$ vs $2.30 \pm 0.64 \mathrm{~mL} / \mathrm{g} / \mathrm{min}$ and $2.21 \pm 0.87$ vs $2.90 \pm 0.90$, respectively, both $p<0.0001)$. There was no difference in rest MBF $(0.79 \pm 0.24$ and $0.82 \pm 0.24$, respectively, $p=0.47$ ).

demographic factors (age, sex), comorbidities (hypertension, diabetes mellitus and dyslipidaemia) and CMR parameters (total LGE, EDV and mass indexed for BSA and EF). Of these, only increasing indexed LV mass was the only factor associated with reduced perfusion $\left(\mathrm{R}^{2}\right.$ for the model $0.186, \mathrm{p}=0.036$, table 2 ) suggesting that reduced perfusion is mainly independent of currently measured parameters.

Analysis was also performed for each myocardial segment. On a per-segment basis, the parameters influencing stress MBF were determined using a multivariable linear regression model taking account within subject dependency. Both the percentage of myocardial late enhancement and wall thickness were associated with impaired MBF per segment $(p<0.001$ for both, table 3). A further segmental analysis was performed comparing stress MBF in apparently 'normal' segments in the HCM cohort to the control group. When only LGE-free segments with a wall thickness $<11 \mathrm{~mm}$ were compared with controls, the stress

Table 2 Multiple linear regression model for the dependent variable global stress myocardial blood flow (MBF). Global stress MBF was independently influenced by indexed left ventricle (LV) mass

\begin{tabular}{|c|c|c|c|c|c|}
\hline & Beta & SE & $\begin{array}{l}95 \% \mathrm{Cl} \text { lower } \\
\text { bound }\end{array}$ & $\begin{array}{l}95 \% \mathrm{Cl} \text { upper } \\
\text { bound }\end{array}$ & $P$ value \\
\hline Constant & 3.317 & 0.939 & 1.439 & 5.184 & 0.001 \\
\hline Age & -0.009 & 0.006 & -0.210 & 0.003 & 0.135 \\
\hline Sex & -0.101 & 0.176 & -0.451 & 0.229 & 0.569 \\
\hline Diabetes & 0.138 & 0.168 & -0.196 & 0.472 & 0.413 \\
\hline Hypertension & -0.136 & 0.130 & -0.396 & 0.123 & 0.299 \\
\hline Dyslipidaemia & -0.264 & 0.161 & -0.584 & 0.057 & 0.106 \\
\hline LVEDVi & 0.003 & 0.006 & -0.009 & 0.014 & 0.653 \\
\hline LVEF & -0.012 & 0.009 & -0.029 & 0.006 & 0.193 \\
\hline LV mass-i & -0.006 & 0.003 & -0.013 & 0.000 & 0.044 \\
\hline LGE & 0.005 & 0.004 & -0.002 & 0.012 & 0.170 \\
\hline
\end{tabular}

$R^{2}=0.186$ for the model, $p=0.036$. $P$ values in bold are statistically significant. $L G E$, late gadolinium enhancement; LVEDVi, left ventricle end-diastolic volume indexed for body surface area (BSA); LVEF, left ventricular ejection fraction; LV mass-i, left ventricle mass indexed for BSA.
Table 3 Mixed effects linear regression model, controlling for withinsubject dependency, for the dependent variable segmental stress myocardial blood flow (MBF)

\begin{tabular}{lclccc}
\hline & & & \multicolumn{2}{c}{$95 \% \mathrm{Cl}$ lower } & $95 \% \mathrm{Cl}$ upper \\
& Beta & SE & bound & bound & P value \\
\hline Intercept & 2.269 & 0.070 & 2.134 & 2.409 & $<0.001$ \\
Wall thickness & -0.050 & 0.004 & -0.060 & -0.043 & $<0.001$ \\
LGE & -0.006 & 0.001 & -0.008 & -0.004 & $<0.001$ \\
\hline
\end{tabular}

Wall thickness and percentage late gadolinium enhancement (LGE) per segment were treated as continuous variables and were independently associated with stress MBF. $P$ values in bold are statistically significant.

MBF remained significantly lower $(1.88 \pm 0.81 \mathrm{~mL} / \mathrm{g} / \mathrm{min}$ vs $2.32 \pm 0.78 \mathrm{~mL} / \mathrm{g} / \mathrm{min}, \mathrm{p}<0.001$ ).

In HCM, stress flow paradoxically lower than rest (MPR $<1$ ) was observed in at least one AHA segment in 21 patients (21\%) (example in figure 1). The finding was more common with increasing wall thickness and LGE (only 1.8\% of segments with MPR $<1$ had normal wall thickness and no LGE). An MPR $<1$ was mainly a subendocardial phenomenon (subendocardial, transmural, subepicardial: $16.3 \%, 5.6 \%$ and $4.7 \%$ of segments) meaning a subendocardial MPR $<1$ occurred in at least one segment in $43(42.6 \%)$ patients.

Segments with visually confluent LGE had a mean stress MBF $1.31 \mathrm{~mL} / \mathrm{g} / \mathrm{min}$, segments with diffuse LGE $1.38 \mathrm{~mL} / \mathrm{g} / \mathrm{min}$, segments with LVH but no LGE $1.48 \mathrm{~mL} / \mathrm{g} / \mathrm{min}$ and segments with no LGE or LVH $1.88 \mathrm{~mL} / \mathrm{g} / \mathrm{min}$. There was no significant difference in the stress MBF of confluent versus diffuse LGE $(p=0.352)$. Confluent LGE had lower stress MBF than LVH segments without LGE $(p=0.015)$ but this did not reach significance for diffuse LGE $(p=0.054)$.

\section{DISCUSSION}

In this largest quantitative CMR perfusion study published in HCM to date, we have shown that microvascular dysfunction is common and somewhat underappreciated in HCM with $78 \%$ of patients having perfusion defects on clinical read. Also, global MBF was low throughout the population. The perfusion abnormalities are not explained by epicardial coronary disease or conventional cardiovascular risk factors, and only partly explained by LGE and hypertrophy, occurring even in the absence of both. Stress MBF is however lowest in the most hypertrophied and fibrotic segments and perfusion can actually fall during stress (giving an MPR $<1$ ). This was a relatively common finding, with over one-fifth of patients having at least one myocardial segment with a lower stress blood flow.

Our results support the previous literature of perfusion in HCM using different modalities over decades. ${ }^{9} 11-14$ 22-24 Initial (non-quantitative) assessment was performed using MPS O'Gara et al in 1987 found visual stress-induced perfusion defects in 41/72 (57\%) patients with HCM. ${ }^{25}$ The perfusion defects seen were independent of patients' symptoms. Furthermore, visual perfusion defects have been shown to be associated with an abnormal blood pressure response to exercise ${ }^{26}$ and to improve with medical therapy with verapamil. ${ }^{27}$ In our study, the per cent of patients with perfusion defect was higher than this at $78 \%$. This may reflect improvements in sensitivity in detection of perfusion defects using latest CMR technology or the fact that this was a clinically referred patient population.

Quantitative perfusion using PET has also investigated MBF in HCM. Camici et al investigated 23 patients with HCM and found that they had impaired perfusion reserve compared with 
a comparator cohort, even in the non-hypertrophied LV-free wall. ${ }^{9}$ There is also prognostic information encoded in MPR as determined by PET and those with impaired perfusion have worse outcomes. ${ }^{11}$

The evidence base for quantitative perfusion CMR is more limited but increasing in recent years. Petersen et al investigated 35 patients with HCM finding impairment in stress MBF correlating with fibrosis and wall thickness predominately affecting the subendocardium. These findings are consistent with what we have found. However, we additionally found that perfusion abnormalities could even be present in segments that were non-hypertrophied and contained no LGE. ${ }^{12} 13$ To our knowledge, this is the largest CMR quantitative perfusion study, with prior cohorts including 30-40 patients and similar to the largest quantitative PET study of 100 patients. ${ }^{28}$

Ismail et al investigated 35 patients with HCM using a pixelwise quantification approach with similar findings. ${ }^{13}$ As well as being a larger cohort, perfusion techniques have developed, here using the single-bolus, dual-sequence approach to overcome arterial input function clipping, automated motion correction requiring no uncomfortable breath holds, inline map reconstruction and automated flow quantification (within $30 \mathrm{~s}$ ) with no user input for global, regional or subsegmental flow data extraction. ${ }^{15}$ Other small HCM cohorts have used either semiquantitative or the dual-sequence approach with broadly consistent results. ${ }^{14} 24$ We found an inverse correlation between MPR or stress MBF and both wall thickness and LGE. However, these associations were relatively modest, and even myocardial segments without LVH or LGE had impaired perfusion, suggesting that microvascular dysfunction is substantially independent of macroscopic scar and hypertrophy, and may be an important marker in early HCM. There have been pathological studies looking at ischaemia in HCM. Basso et al looked at 19 patients with HCM with SCD. ${ }^{29}$ They found abnormal intramural small vessels in the myocardium and evidence of all stages of ischaemia from acute to chronic appearances even in the absence of epicardial coronary artery disease, and postulated that HCM replacement fibrosis is triggered by myocardial ischaemia with combination of disarray and ischaemia being the arrhythmic substrate. In another autopsy study of 72 patients Varnava et al found a poor correlation between small vessel disease and LVH and there was evidence of small vessel disease even in children. ${ }^{5}$

A striking finding was that stress flow in HCM could be paradoxically lower than rest in HCM. A perfusion reserve $<1$ has previously been observed and thought to be related exclusively to scar ${ }^{14}$ or modelling artefact. Here, it mostly occurred in the endocardial layer (16.3\% of all myocardial segments) and was associated with increased LGE and wall thickness. However, LGE and hypertrophy cannot fully explain the phenomenon and a small per cent $(1.8 \%$ transmural or $6.9 \%$ subendocardial segments) of this MPR $<1$ segment was free from LGE and LVH. There are multiple possible explanations for perfusion falling during vasodilator stress. Macroscopic steal appears unlikely as the areas of hypoperfusion are large but microscopic steal remains possible. Another explanation would be an altered myocardial vasomotor response to adenosine in HCM. The vascular response to adenosine is organ specific (eg, splenic vasoconstriction, myocardial vasodilatation) and modification of the myocardium in disease could play a role. Another possibility would include a mechanical explanation with prolonged regional systole and altered myocardial mechanics secondary to a vasodilatation-induced tachycardia inducing perfusion defects. ${ }^{7}$

In this study we have demonstrated a transmural perfusion gradient in subjects at stress, where the subendocardial perfusion is reduced relative to the subepicardium. This is consistent with previous microsphere studies using animal models ${ }^{3031}$ and CMR studies in health and disease. ${ }^{32}{ }^{33}$ In contrast, PET studies have suggested that at stress the subendocardium is more highly perfused than the subepicardium. ${ }^{34} 35$ Possible explanations include differences in the spatial resolution of the techniques or other method-specific factors which influence the perfusion data for PET and CMR.

Our study is limited by the fact that our patient population was clinically referred for scans which may put them into a higher risk category. Additionally, all were recruited from a tertiary centre that includes specialist cardiomyopathy services. Similar to other studies, there was no tissue correlation and our cohort was not designed for prognostic endpoints. Vasodilator stress demonstrates areas of hypoperfusion and is one step remote from ischaemia but the link between these concepts has been made previously.

In conclusion, using fully quantitative CMR perfusion mapping incorporated into a clinical workflow, we have demonstrated the role of microvascular dysfunction in HCM and that while this is statistically strongly associated with regional hypertrophy and fibrosis, these processes explain only a small amount of myocardial stress blood flow heterogeneity. Flow was noted to actually fall during vasodilator stress and can be abnormal even in remote (no LVH, no LGE) myocardium suggesting microvascular dysfunction may occur early in phenotype development. CMR perfusion mapping is a useful new tool to investigate the pathophysiology of cardiomyopathy, making evaluating ischaemia a testable SCD risk factor and a potential therapeutic target.

Key messages

What is already known on this subject?

- Hypertrophic cardiomyopathy (HCM) is a common genetic cardiomyopathy in which there is myocyte disarray, left ventricular hypertrophy, small vessel disease and hypertrophy.

\section{What might this study add?}

- This study has shown that microvascular dysfunction is common in HCM and worsens with increasing disease severity (hypertrophy and fibrosis). Perfusion can actually fall with vasodilator stress and can be abnormal even in apparently normal myocardium suggesting it may occur early in the disease process.

How might this impact on clinical practice?

- Quantitative perfusion cardiovascular magnetic resonance is becoming routine in clinical practice and may be useful in detecting early disease in $\mathrm{HCM}$, could act as a testable risk factor for sudden cardiac death and be a potential therapeutic target.

Acknowledgements This work using the Barts BioResource forms part of the research areas contributing to the translational research portfolio of the Biomedical Research Centre at Barts which is supported and funded by the National Institute for Health Research. Similar Biomedical Research Centre support is via University College London Hospitals also funded by the National Institute for Health Research.

Contributors CC and KDK contributed equally to this work. CC, KDK and JCM drafted the article, and contributed to the conception and design of the work and final approval of the version to be published. CC, KDK, JBA and AS contributed to data collection. CC, KDK, SR, FR, RB, HX and PK contributed to the data analysis and interpretation. HX, RH, GC, LRL, LAEB, CM, SEP, SP, PK and SAM contributed to the critical revision of the article. 
Funding This study was supported by research grant from the European Association of Cardiovascular Imaging (to CC).

Competing interests SEP provides consultancy to Circle Cardiovascular Imaging (Calgary, Alberta, Canada).

Patient consent for publication Not required.

Provenance and peer review Not commissioned; externally peer reviewed.

Data availability statement Data are available upon reasonable request.

Open access This is an open access article distributed in accordance with the Creative Commons Attribution 4.0 Unported (CC BY 4.0) license, which permits others to copy, redistribute, remix, transform and build upon this work for any purpose, provided the original work is properly cited, a link to the licence is given, and indication of whether changes were made. See: https://creativecommons.org/ licenses/by/4.0/

\section{ORCID iDs}

Kristopher D Knott http://orcid.org/0000-0003-1611-817X

Louise Anne Elizabeth Brown http://orcid.org/0000-0002-1327-6482

\section{REFERENCES}

1 Alcalai R, Seidman JG, Seidman CE. Genetic basis of hypertrophic cardiomyopathy: from bench to the clinics. J Cardiovasc Electrophysiol 2008;19:104-10.

2 Elliott PM, Anastasakis A, Borger MA, et al. 2014 ESC guidelines on diagnosis and management of hypertrophic cardiomyopathy: the task force for the diagnosis and management of hypertrophic cardiomyopathy of the European Society of cardiology (ESC). Eur Heart J 2014:35:2733-79.

3 Elliott PM, Kaski JC, Prasad K, et al. Chest pain during daily life in patients with hypertrophic cardiomyopathy: an ambulatory electrocardiographic study. Eur Heart J 1996;17:1056-64

4 Finocchiaro G, Papadakis M, Robertus J-L, et al. Etiology of sudden death in sports: insights from a United Kingdom regional registry. J Am Coll Cardiol 2016;67:2108-15

5 Varnava AM, Elliott PM, Sharma S, et al. Hypertrophic cardiomyopathy: the interrelation of disarray, fibrosis, and small vessel disease. Heart 2000;84:476-82.

6 Maron MS, Olivotto I, Maron BJ, et al. The case for myocardial ischemia in hypertrophic cardiomyopathy. J Am Coll Cardiol 2009;54:866-75.

7 Stephenson E, Monney P, Pugliese F, et al. Ineffective and prolonged apical contraction is associated with chest pain and ischaemia in apical hypertrophic cardiomyopathy. Int J Cardiol 2018;251:65-70.

8 Mohiddin SA, Begley D, Shih J, et al. Myocardial bridging does not predict sudden death in children with hypertrophic cardiomyopathy but is associated with more severe cardiac disease. J Am Coll Cardiol 2000;36:2270-8.

9 Camici P, Chiriatti G, Lorenzoni $R$, et al. Coronary vasodilation is impaired in both hypertrophied and nonhypertrophied myocardium of patients with hypertrophic cardiomyopathy: a study with nitrogen-13 ammonia and positron emission tomography. J Am Coll Cardiol 1991;17:879-86.

10 Yamada Met al. Dipyridamole stress thallium-201 perfusion abnormalities in patients with hypertrophic cardiomyopathy. Relationship to clinical presentation and outcome. Eur Heart J 1998;19:500-7.

11 Cecchi F, Olivotto I, Gistri R, et al. Coronary microvascular dysfunction and prognosis in hypertrophic cardiomyopathy. N Eng/ J Med 2003;349:1027-35

12 Petersen SE, Jerosch-Herold M, Hudsmith LE, et al. Evidence for microvascular dysfunction in hypertrophic cardiomyopathy: new insights from multiparametric magnetic resonance imaging. Circulation 2007;115:2418-25.

13 Ismail TF, Hsu L-Y, Greve AM, et al. Coronary microvascular ischemia in hypertrophic cardiomyopathy - a pixel-wise quantitative cardiovascular magnetic resonance perfusion study. J Cardiovasc Magn Reson 2014;16.

14 Villa ADM, Sammut E, Zarinabad N, et al. Microvascular ischemia in hypertrophic cardiomyopathy: new insights from high-resolution combined quantification of perfusion and late gadolinium enhancement. J Cardiovasc Magn Reson 2016;18.
15 Kellman $\mathrm{P}$, Hansen MS, Nielles-Vallespin S, et al. Myocardial perfusion cardiovascular magnetic resonance: optimized dual sequence and reconstruction for quantification. $J$ Cardiovasc Magn Reson 2017;19.

16 Hansen MS, Sørensen TS. Gadgetron: an open source framework for medical image reconstruction. Magn Reson Med 2013;69:1768-76.

17 Engblom $\mathrm{H}$, Xue $\mathrm{H}$, Akil S, et al. Fully quantitative cardiovascular magnetic resonance myocardial perfusion ready for clinical use: a comparison between cardiovascular magnetic resonance imaging and positron emission tomography. J Cardiovasc Magn Reson 2017;19.

18 Knott KD, Camaioni C, Ramasamy A, et al. Quantitative myocardial perfusion in coronary artery disease: a perfusion mapping study. J Magn Reson Imaging 2019;50:756-62.

19 Kotecha T, Martinez-Naharro A, Boldrini M, et al. Automated Pixel-Wise Quantitative Myocardial Perfusion Mapping by CMR to Detect Obstructive Coronary Artery Disease and Coronary Microvascular Dysfunction: Validation Against Invasive Coronary Physiology. JACC Cardiovasc Imaging 2019;12:1958-69.

20 Knott KD, Augusto JB, Nordin S, et al. Quantitative myocardial perfusion in Fabry disease. Circ Cardiovasc Imaging 2019;12:e008872.

21 Cerqueira MD, Weissman NJ, Dilsizian V, et al. Standardized myocardial segmentation and nomenclature for tomographic imaging of the heart. A statement for healthcare professionals from the cardiac imaging Committee of the Council on clinical cardiology of the American heart association. Int J Cardiovasc Imaging 2002;18:539-42

22 Sotgia B, Sciagrà R, Olivotto I, et al. Spatial relationship between coronary microvascular dysfunction and delayed contrast enhancement in patients with hypertrophic cardiomyopathy. J Nucl Med 2008:49:1090-6.

23 Yin L, Xu H-Y, Zheng S-S, et al. 3.0 T magnetic resonance myocardial perfusion imaging for semi-quantitative evaluation of coronary microvascular dysfunction in hypertrophic cardiomyopathy. Int I Cardiovasc Imaging 2017;33:1949-59.

24 Tezuka D, Kosuge H, Terashima M, et al. Myocardial perfusion reserve quantified by cardiac magnetic resonance imaging is associated with late gadolinium enhancement in hypertrophic cardiomyopathy. Heart Vessels 2018;33:513-20.

25 O'Gara PT, Bonow RO, Maron BJ, et al. Myocardial perfusion abnormalities in patients with hypertrophic cardiomyopathy: assessment with thallium-201 emission computed tomography. Circulation 1987:76:1214-23.

26 Yoshida N, Ikeda H, Wada T, et al. Exercise-Induced abnormal blood pressure responses are related to subendocardial ischemia in hypertrophic cardiomyopathy. $J$ Am Coll Cardiol 1998;32:1938-42.

27 Udelson JE, Bonow RO, O'Gara PT, et al. Verapamil prevents silent myocardial perfusion abnormalities during exercise in asymptomatic patients with hypertrophic cardiomyopathy. Circulation 1989;79:1052-60.

28 Castagnoli H, Ferrantini C, Coppini R, et al. Role of quantitative myocardial positron emission tomography for risk stratification in patients with hypertrophic cardiomyopathy: a 2016 reappraisal. Eur J Nucl Med Mol Imaging 2016;43:2413-22.

29 Basso C, Thiene G, Corrado D, et al. Hypertrophic cardiomyopathy and sudden death in the young: pathologic evidence of myocardial ischemia. Hum Pathol 2000;31:988-98

30 Cobb FR, Bache RJ, Greenfield JC. Regional myocardial blood flow in awake dogs. J Clin Invest 1974;53:1618-25.

31 Leppo JA, Simons M, Hood WB. Effect of adenosine on transmural flow gradients in normal canine myocardium. J Cardiovasc Pharmacol 1984;6:1115-9.

32 Fairbairn TA, Motwani M, Mather AN, et al. Cardiac MR imaging to measure myocardial blood flow response to the cold pressor test in healthy smokers and nonsmokers. Radiology 2014;270:82-90.

33 Chiribiri A, Hautvast GLTF, Lockie T, et al. Assessment of coronary artery stenosis severity and location: quantitative analysis of transmural perfusion gradients by highresolution MRI versus FFR. JACC Cardiovasc Imaging 2013:6:600-9.

34 Vermeltfoort IA, Raijmakers PG, Lubberink M, et al. Feasibility of subendocardial and subepicardial myocardial perfusion measurements in healthy normals with (15) 0-labeled water and positron emission tomography. J Nucl Cardiol 2011;18:650-6.

35 Sciagrà R, Milan E, Giubbini R, et al. Sub-endocardial and sub-epicardial measurement of myocardial blood flow using 13NH3 PET in man. Journal of Nuclear Cardiology 2018;3. 